\title{
Effect of Weak Magnetic Field Treatment on Optical Properties of Epitaxial GaN Films
}

\author{
R.A. Red'ko ${ }^{*}, 1$, R.V. Konakova ${ }^{1}$, V.V. Milenin ${ }^{1}$, S.M. Red'ko ${ }^{1}$, V.V. Shvalagin ${ }^{2}$, N.S. Zayats ${ }^{1}$ and \\ Yu. N. Sveshnikov ${ }^{3}$ \\ ${ }^{I}$ V. Lashkaryov Institute of Semiconductor Physics of the National Academy of Sciences of Ukraine, 41 Nauky Pr., \\ 03028 Kyiv, Ukraine \\ ${ }^{2}$ L. Pysarzhevskiy Institute of Physical Chemistry of the National Academy of Sciences of Ukraine, 31 Nauky Pr., 03028 \\ Kyiv, Ukraine \\ ${ }^{3}$ Elma-Malachite JSC, 124460 Zelenograd, Russia
}

\begin{abstract}
The results of optical studies of the effect of pulsed magnetic field ( $B=60 \mathrm{mT}, \tau=1.2 \mathrm{~ms}, t=5 \mathrm{~min})$ on the structural perfection and recombination properties of epitaxial $\mathrm{GaN}$ films grown on sapphire are presented. Nonmonotonic changes in the spectral dependence of transmittance and photoluminescence were detected for GaN films after exposure to magnetic field. They occurred during several days, with a maximum deviation from the initial values on the 12 th day. The observed effect is presumably related to the magnetic field-induced changes in parameters of the surfaces and interfaces of epitaxial films.
\end{abstract}

Keywords: Optical transmittance, photoluminescence, weak magnetic field.

\section{INTRODUCTION}

It is well known that epitaxial layers of gallium nitride grown on classical sapphire substrate by metal organic chemical vapor deposition (MOCVD) technique have many electrically active defects of different nature that are non-uniformly distributed in the grown layers. To improve structural perfection of epitaxial layers, various technological approaches usually based on high temperature procedures were used in the course of growth processes. They led to complication of the epitaxial structure technology, but required quality has not been always achieved. To change the structural characteristics of epitaxial films (EFs), not only improving of the growth process can be used but different external actions on the grown layers as well. Among the latter is magnetic field (MF) treatment. It was established [1] that weak MF can affect the structure-sensitive physical processes in various semiconductor materials, even at room temperature.

This feature has formed the ground for (i) study of optical properties changes in GaN EFs stimulated by weak MF and (ii) identification of structural elements that are sensitive to the effects of weak MF.

\section{EXPERIMENT}

We investigated GaN EFs (thickness of $\sim 2 \mu \mathrm{m}$ ) MOCVD-grown on a sapphire substrate $(\sim 430 \mu \mathrm{m})$. A total

*Address correspondence to this author at the V. Lashkaryov Institute of Semiconductor Physics of the National Academy of Sciences of Ukraine, 41 Nauky Pr., 03028 Kyiv, Ukraine; Tel: (044) 525-61-82;

E-mails: konakova@isp.kiev.ua, redko.rom@gmail.com of 50 samples have been investigated. The charge carrier concentration of $\sim 8 \cdot 10^{17} \mathrm{~cm}^{-3}$ was determined by using plasma resonance.

Photoluminescence (PL) spectra of the samples illuminated by light with $h v=3.94 \mathrm{eV}$ were measured in the 1.9-3.5 eV spectral range at $300 \mathrm{~K}$ using a spectrometer (Perkin-Elmer LS55 PL). So we could study band-to-band as well as impurity PL.

The spectra of optical transmittance (OT) were measured at $300 \mathrm{~K}$ with a spectrophotometer (Specord 210) in the 2.0$3.5 \mathrm{eV}$ spectral range. The treatment of the material under investigation was performed at room temperature by weak pulsed MF with $B=60 \mathrm{mT}, \tau=1.2 \mathrm{~ms}, t=5 \mathrm{~min}$.

The magnetic induction $B$ was normal to the sample surface. For PL and OT measurements, we used a standard normalization methodology of the received signal with the spectrum of the reference sample. As much as $1 / 3$ of the surface of tested sample formed optical signal, while the rest was covered with a light-tight material.

The reference (not treated) films served as an indicator of natural aging of samples and were studied along with those exposed to MF. Between the measurements, they were kept under the same conditions as the treated samples. The exciting light beam at PL (OT) measurements was oriented at an angle of $45^{\circ}\left(0^{\circ}\right)$ to the surface, respectively.

\section{RESULTS AND DISCUSSION}

All samples under investigation demonstrated similar results. We have chosen the most typical ones, but general features were inherent for all subjects studied. The PL 
spectrum of $\mathrm{GaN}$ EFs measured at room temperature consisted of two bands peaked at about 3.42 and $2.34 \mathrm{eV}$ (Fig. 1, the initial curve). The spectrum was typical for $n$ GaN films (doped with different donors and undoped) [2] grown using different technologies.

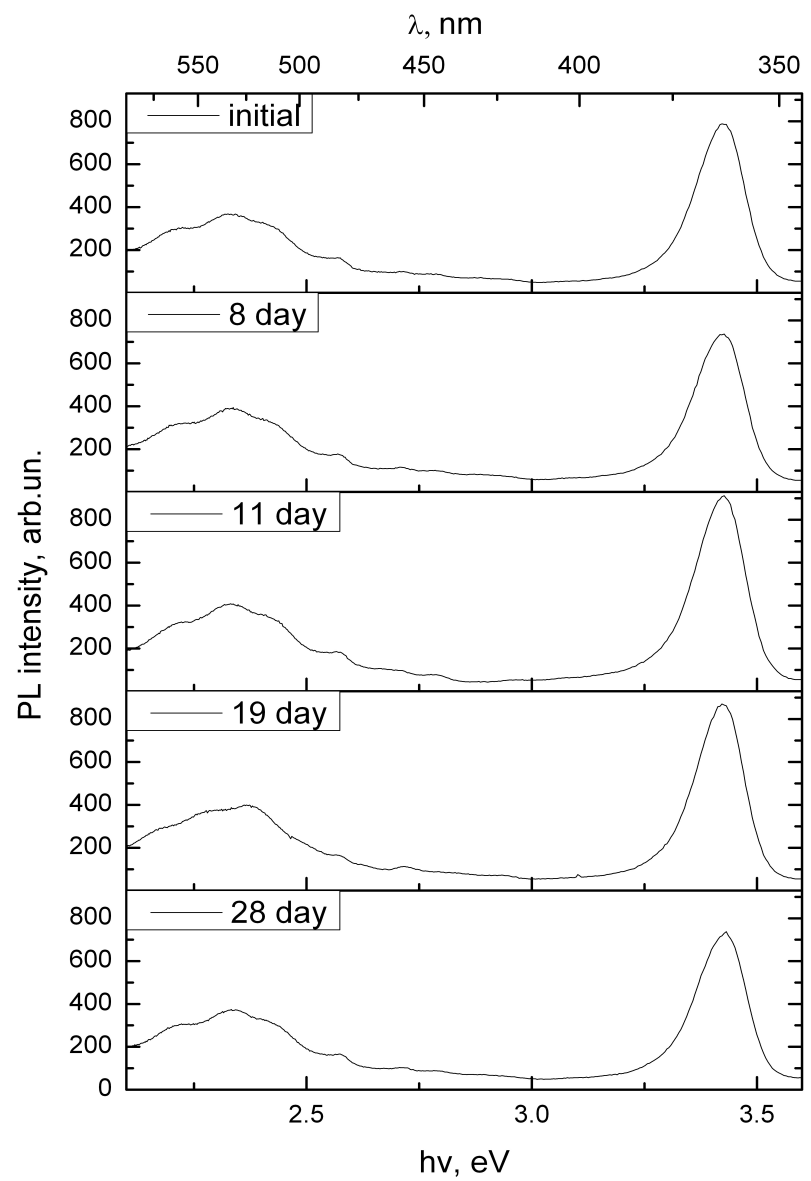

Fig. (1). The dependence of integral PL of $\mathrm{GaN} / \mathrm{Al}_{2} \mathrm{O}_{3}$ on the time after MF treatment.

The more intense band in the PL spectrum could be related to radiative recombination of excitons [3]. It is considered that radiative recombination of excitons bound to neutral impurities is negligible at temperatures $\sim 300 \mathrm{~K}$, while the main contribution to the observed band comes from free excitons recombination $\left(E_{f e}\right.$ is about $\left.30 \mathrm{meV}\right)$.

The second band present in the PL spectrum at $2.34 \mathrm{eV}$ that is apparently not trivial and has a large width $(\sim 350 \mathrm{meV})$ could be associated with defects in the film (yellow luminescence) $[2$, $3]$. The origin of this emission line is assigned to impurityvacancy complexes. It is due to increase in intensity of this band in the presence of dislocations [2, 4].

The results of OT experimental investigations are in good agreement with optical studies of GaN EFs performed earlier in [5] and presented in Fig. (2). Typical oscillations are observed at energies smaller than band gap energy. This is due to interference that occurs at light reflection from the $\mathrm{GaN} / \mathrm{Al}_{2} \mathrm{O}_{3}$ interface. Presence of interference allows determination of refractive index $n$ for GaN film and (as $n$ is known) the epitaxial layer thickness $d[6]$.

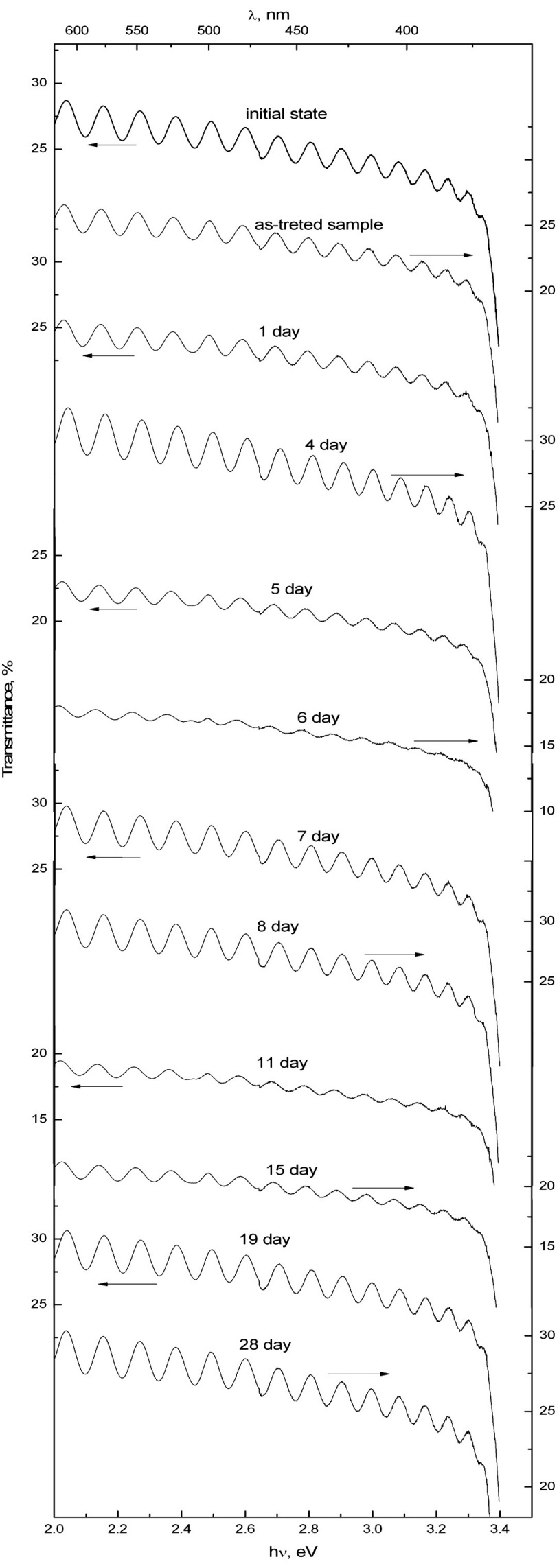

Fig. (2). The dependence of OT for epitaxial $\mathrm{GaN} / \mathrm{Al}_{2} \mathrm{O}_{3}$ structure on the time after MF treatment. 
The effect of MF treatment on PL spectra is shown in Fig. (1). Some features in the PL spectral dependences should be noted:

i) changes in the PL spectra are observed only after a certain time interval after treatment $(\sim 7$ days $)$ and continue for a long period at samples aging under laboratory conditions;

ii) the energy position of the near-edge PL band does not change in the course of long time relaxation. Shifting of the impurity band maximum position to the red spectral region is accompanied with a small change of its form. The maximal shift $(\sim 50 \mathrm{meV})$ was registered after aging for 19 days;

iii) the long-term changes in the intensities of near-edge and impurity PL bands in the course of relaxation were nonmonotonic.

Fig. (3) shows the changes in the band intensities normalized to the corresponding initial values. The observed changes are symbate. However, MF treatment affects differently the intensities of the near-edge and impurity bands. The most essential changes were detected for the near-edge band (the intensity increased by $20-25 \%$ ). For the impurity band, a change of intensity was much weaker, with smoother reduction to the initial value.

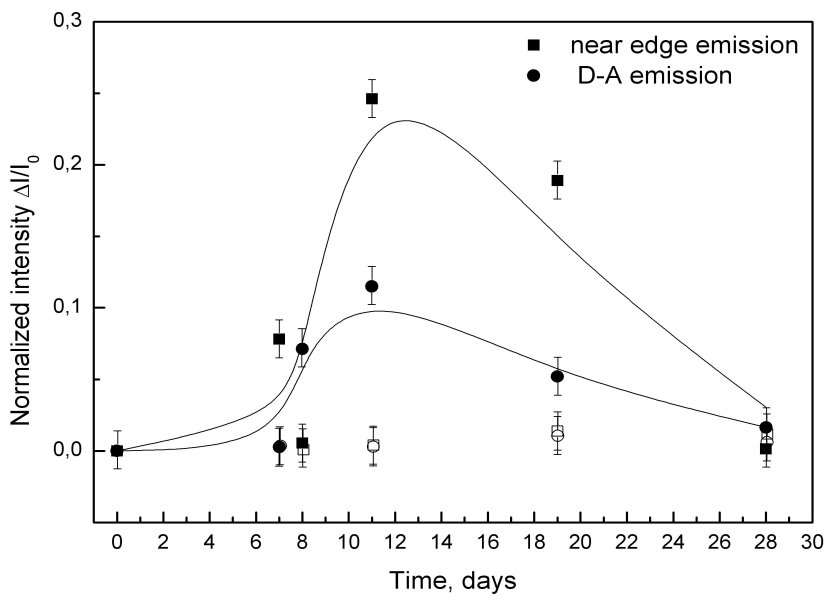

Fig. (3). The time dependence of observed PL bands intensities (marks - experiment, curve - averaging, empty squares and circlescontrol sample).

Let us try to consider factors caused by weak MF treatment that can affect the radiative recombination in the structures under investigation. Strongly absorbed light $\left(\lambda=315 \mathrm{~nm}\right.$, absorption coefficient $\alpha=1.25 \cdot 10^{5} \mathrm{~cm}^{-1}$ [7] $)$ was used to excite PL. Thus, the thickness of the layer where electron-hole pairs are generated is $\sim 8 \cdot 10^{-6} \mathrm{~cm}$. On the other hand, it is possible to estimate the space charge region length $L$ (the Schottky layer width) from the expression [8]:

$L=\left(\frac{2 \varepsilon \varepsilon_{0} \phi_{0}}{e n_{0}}\right)^{1 / 2}$,

where $\varphi_{0}$ is band bending, $n_{0}$ electron concentration; the rest of parameters are well-known. For our estimation, we took $\varphi_{0}=1 \mathrm{eV}[2]$ and $n_{0}=\sim 8 \cdot 10^{17} \mathrm{~cm}^{-3}$. Assuming a uniform distribution of impurities, we obtained for Schottky layer width $\sim 4.04 \cdot 10^{-6} \mathrm{~cm}$. Thus, the light absorption depth and Schottky layer width are comparable, so changes in the physical and chemical state of the surface should affect the intensities of different PL bands in GaN.

The effect of surface electronic structure on radiative recombination was analyzed in $[9,10]$. As was noted in [10], the change in the surface band bending is accompanied by nonmonotonic variation of PL bands intensities, with a clear selectivity for different bands, just as was observed in our case.

The main feature of observed phenomena is small energy value $\left(\sim 10^{-5} \mathrm{eV}\right)$ obtained in the magnetic field $\left(\mu_{B} B<<k T\right.$, $\mu_{B}$ is the Bohr magneton). According to the traditional approach, this energy should be much higher than that of thermal fluctuations of electron gas at room temperature. The effect of electromagnetic induction can appear in semiconductor materials at weak MF treatment. Its value can be estimated from the well-known relation $\varepsilon=-\frac{\partial \Phi}{\partial t}$, where $\Phi=\int_{S} B_{n} d S$. For $B=60 \mathrm{mT}, \tau=1.2 \mathrm{~ms}$ and $S \sim 1 \mathrm{~cm}^{2}$, we have $\varepsilon \approx 5 \mathrm{mV}$. Obviously, such voltage is not able to induce any structural modifications. The most likely mechanism of weak MF action is associated with breakage of strained chemical bonds at the surface and in the impuritydefect complexes in the near-surface region due to MFinduced intercombination transitions. So weak MF treatment can lead to changing of the electronic structure of the surface and appearance of charged defects, thus resulting in modification of the luminescence spectra. Moreover, according to [11-13], these changes in the PL spectra should be long-term and nonmonotonic.

On the other hand, MF-stimulated oxidation can be a factor influencing the PL bands [12]. This can change the elastically-strained state of the GaN surface region. A shift of the maximum, strong PL quenching and broadening of the edge band due to increase of native stresses in $\mathrm{GaN} / \mathrm{Al}_{2} \mathrm{O}_{3}$ were observed in [14], while the yellow band intensity did not change essentially.

The qualitatively different changes of observed PL bands in the same crystal indicate more than one factor of influence. For example, a decrease (increase) in the rate of surface nonradiative recombination could be another reason for the changes observed. So, we should believe that the observed modification of PL spectra occurs due to the action of factors considered above.

While the results of PL measurements indicate variation of the defect concentration (and energy state) in the surface layer, the OT spectral curves depend on the defect concentration in the GaN film and its thickness.

The OT spectra of $\mathrm{GaN} / \mathrm{Al}_{2} \mathrm{O}_{3}$ taken during 30 days after weak MF treatment are shown in Fig. (2). It can be seen that there are long-term nonmonotonic changes of transmittance. Moreover, the transformation of curves obtained, as well as those at PL measurements, does not occur immediately after 
MF treatment. Changes in the peak heights and distances between peak positions were detected.

Using the method of interference analysis proposed in [6], it is possible to estimate the effective thickness $d$ for $\mathrm{GaN}$ EFs from the expression

$$
d=\frac{\lambda_{1} \lambda_{2}}{2 n\left(\lambda_{2}-\lambda_{1}\right)},
$$

where $\lambda_{1}$ and $\lambda_{2}$ are the wavelengths of neighboring interference maxima and $n$ the $\mathrm{GaN}$ refractive index $(n \approx 2.4$ [15]). The dependence $d(t)$ obtained under the assumption of constancy of $n$ at MF treatment is shown in Fig. (4). The presented data indicate variation in the macrogeometry of structure under investigation.

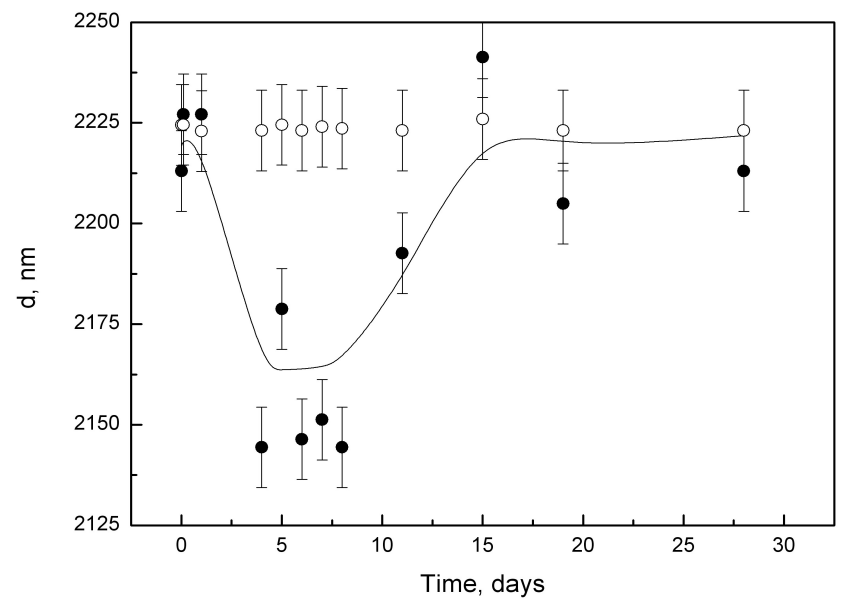

Fig. (4). The variation of thickness of the layer that formed interference as a function of time after weak MF treatment (filled circles - experiment, line - averaging, empty circles - control sample).

Large mismatch between the film and substrate lattice constants results in strong tensile stresses at the interface. Their compensation is provided not only by generation of misfit dislocations but also by diffusion broadening of the interface due to intermixing of film and substrate atomic components, as well as by possible formation of new phases, such as amorphous oxynitride. As a result, a structurally inhomogeneous transition region (TR) of complex chemical composition appears. Such TR could affect the interference conditions for reflected (transmitted) light.

Indeed, if TR thickness is large enough, then interference occurs due to light reflection from the $\mathrm{GaN}$ film-TR interface. But if TR thickness is too small (TR is transparent to incident radiation), then reflection occurs from the TRsubstrate interface. In the intermediate case, the interference changes because of variation of TR contribution which may cause the observed nonmonotonic behavior of the curve in Fig. (4).

The physical reason for TR transformation may be the action of mechanical force that appears as the structure is subjected to MF. Since magnetization gradient is maximal in the region of EF-substrate interface, the mechanical strength may vary the native stresses at the interface and, consequently, result in appearance of diffusion fluxes of atoms and structural defects that can change TR thickness and structure.

After MF removal, the TR thickness relaxes to its initial value because the reason for native stresses in the structure under investigation is not removed.

\section{CONCLUSION}

Thus, the effect of pulse MF results in structural changes of the surface as well as $\mathrm{GaN}$ film-substrate interface. These changes are long-term and nonmonotonic. MF acts on spins of electrons that take part in the formation of strained bonds on the GaN surface. This results in their weakening with further breakage. Then the surface activation and a change of the surface charge (band bending) occur. The magnitude and sign of surface band bending affect the intensity of PL bands. These facts can also occur at the film-substrate interface, resulting in its structural reorganization; besides, the mechanical forces generated by MF vary its macrogeometry. We expect that scanning electron microscopy and energy dispersive $\mathrm{X}$-ray spectroscopy will confirm our conclusions. MF action can be applied as a costeffective method for modification of the recombination and structural parameters of multilayer EFs and detection of nonequilibrium centers in finished semiconductor materials and devices.

\section{CONFLICT OF INTEREST}

The authors confirm that this article content has no conflict of interest.

\section{ACKNOWLEDGEMENTS}

Declared none.

\section{REFERENCES}

[1] Morgunov RB. Spin micromechanics in the physics of plasticity. Phys Usp 2004; 47: 125

[2] Reshchikov MA, Morkoç H. Luminescence properties of defects in GaN. J Appl Phys 2005; 97: 061301.

[3] Sheu JK, Chi GC. The doping process and dopant characteristics of GaN. J Phys: Condens Matter 2002; 14: R657.

[4] Jeong MS, Kim Y-W, White JO, et al. Spatial variation of photoluminescence and related defects in $\mathrm{InGaN} / \mathrm{GaN}$ quantum wells. Appl Phys Lett 2001; 79: 3440.

[5] Zayats NS, Gentsar PO, Boiko VG, et al. Optical properties of the Si-doped $\mathrm{GaN} / \mathrm{Al}_{2} \mathrm{O}_{3}$ films. Semiconductors 2009; 43: 590 .

[6] Clark AH. Optical properties of polycrystalline semiconductor film. In: Kazmerski LL. Polycrystalline and amorphous thin films and devices. New York: Academic Press 1980.

[7] $\mathrm{Yu} \mathrm{G}$, Wang G, Ishikawa $\mathrm{H}$, et al. Optical properties of wurtzite structure $\mathrm{GaN}$ on sapphire around fundamental absorption edge $(0.78-4.77 \mathrm{eV})$ by spectroscopic ellipsometry and the optical transmission method. Appl Phys Lett 1997; 70: 3209.

[8] Sze SM. Physics of semiconductor devices, A Wiley Interscience Publication. John Wiley \& Sons: New York-Chichester-BrisbaneToronto-Singapore 1981; vol. 1.

[9] Zinets OS, Peka GP, Karkhanin YI. Some problems in the theory of luminescent field effect. Sov Phys Solid State 1964; 6: 3515.

[10] Ermolovich IB, Peka GP, Sheinkman MK. Effect of surface states on luminescent properties of CdS single crystals. Surface Sci 1971; 24: 229. 
[11] Levin MN, Tatarintsev AV, Kostsova OA, Kostsov AM. Activation of a semiconductor surface by a pulsed magnetic field. Tech Phys 2003; 48: 1304

[12] Levin MN, Semenova GV, Tatarintsev AV, Shumskaya ON. The effect of treatment in a pulsed magnetic field on the kinetics of oxidation of indium phosphide crystals. Tech Phys Lett 2005; 31: 762 .
[13] Morgunov RB, Buchachenko AL. Magnetoplasticity and magnetic memory in diamagnetic solids. JETP 2009; 109: 434.

[14] Junaid M, Lundin D, Palisaitis J, et al. Two-domain formation during the epitaxial growth of $\mathrm{GaN}(0001)$ on $c$-plane $\mathrm{Al}_{2} \mathrm{O}_{3}(0001)$ by high power impulse magnetron sputtering. J Appl Phys 2011; 110: 123519 .

[15] Schubert FE. Light-Emitting diodes. $2^{\text {nd }}$ ed. Cambridge University Press 2006.

(C) Red'ko et al.; Licensee Bentham Open.

This is an open access article licensed under the terms of the Creative Commons Attribution Non-Commercial License (http: //creativecommons.org/licenses/by$\mathrm{nc} / 3.0 /$ ) which permits unrestricted, non-commercial use, distribution and reproduction in any medium, provided the work is properly cited. 